\title{
Rutherford backscattering spectrometry analysis of InGaAs nanostructures
}

Grazia Laricchiuta, Wilfried Vandervorst, Ian Vickridge, Matej Mayer, and Johan Meersschaut

Citation: Journal of Vacuum Science \& Technology A 37, 020601 (2019); doi: 10.1116/1.5079520

View online: https://doi.org/10.1116/1.5079520

View Table of Contents: https://avs.scitation.org/toc/jva/37/2

Published by the American Vacuum Society

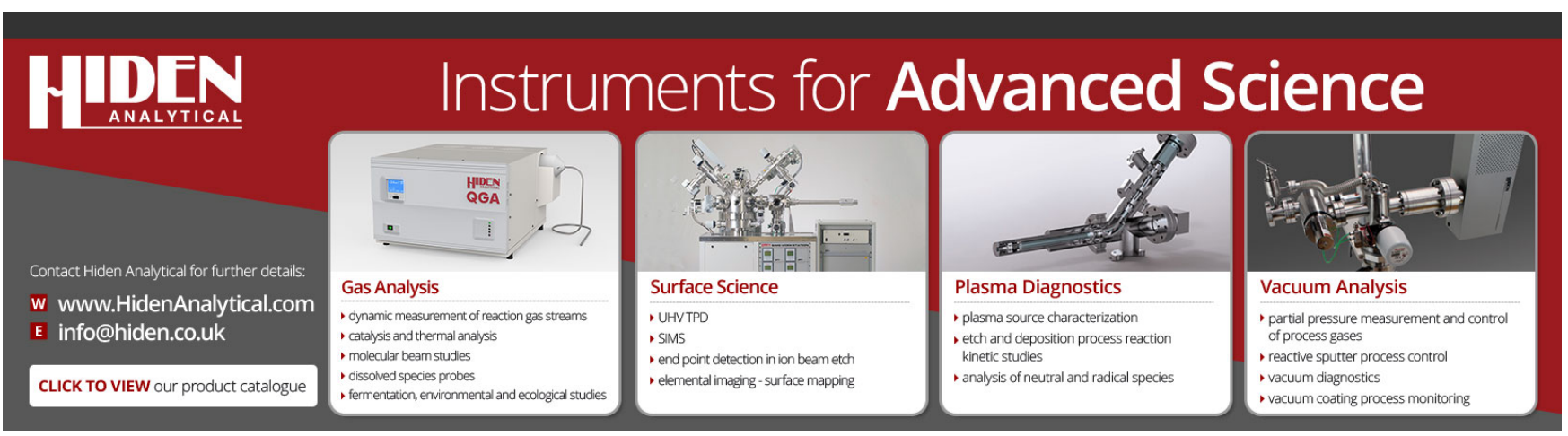




\title{
LETTERS
}

\section{Rutherford backscattering spectrometry analysis of InGaAs nanostructures}

\author{
Grazia Laricchiuta, ${ }^{1,2, a)}$ Wilfried Vandervorst, ${ }^{1,2}$ Ian Vickridge, ${ }^{3}$ Matej Mayer, ${ }^{4}$ \\ and Johan Meersschaut ${ }^{1,2}$ \\ ${ }_{1}^{1}$ IKS, KU Leuven, Celestijnenlaan 200D, B-3001 Leuven, Belgium \\ ${ }^{2}$ Imec, Kapeldreef 75, B-3001 Leuven, Belgium \\ ${ }^{3}$ INSP, Sorbonne Universités, Place Jussieu 4, 75005 Paris, France \\ ${ }^{4}$ Max-Planck-Institut für Plasmaphysik, Boltzmannstr. 2, 85748 Garching, Germany
}

(Received 31 October 2018; accepted 2 January 2019; published 17 January 2019)

\begin{abstract}
In this work, the authors demonstrate that Rutherford backscattering spectrometry (RBS) can be extended from a metrology concept applied to blanket films toward a method to analyze confined nanostructures. By a combination of measurements on an ensemble of devices and extensive simulations, it is feasible to quantify the composition of InGaAs nanostructures $(16-50 \mathrm{~nm})$ embedded periodically in an $\mathrm{SiO}_{2}$ matrix. The methodology is based on measuring multiple fins simultaneously while using the geometrical shape of the structures, obtained from a transmission electron microscopy analysis, as input for a multitude of trajectory calculations. In this way, the authors are able to reproduce the RBS spectra and to demonstrate the sensitivity of the RBS spectra to the quantitative elemental composition of the nanostructures and to variations of their shape and mean areal coverage down to one nanometer. Thus, the authors establish RBS as a viable quantitative characterization technique to probe the composition and structure of periodic arrays of nanostructures. Published by the AVS. https://doi.org/10.1116/1.5079520
\end{abstract}

\section{INTRODUCTION}

The further advancement of the semiconductor technologies, targeting integrated circuits with improved device performance or reduced fabrication costs, requires the exploration of novel geometries, e.g., 3d-finfets, as well as the introduction of new materials such as III-V compound semiconductors. In particular, indium gallium arsenide compounds, for their superior electrical and optical properties, have been considered as a candidate for $\mathrm{n}$-channel MOS devices beyond the $12 \mathrm{~nm}$ technology node ${ }^{1}$ and for optoelectronics applications. ${ }^{2-4}$ However, as these III-V compounds have much larger lattice parameters as compared to $\mathrm{Si}$, their integration on a common Si platform is challenging. Indeed, due to the lattice mismatch, the direct epitaxy of III-V layers on Si could suffer from the creation of various crystalline defects at the interface which, as they normally propagate to the top surface, will strongly degrade the device performances. One way to reduce this propagation is by growing the layers in small features with a high aspect ratio $(>2)$, the concept is commonly referred to as aspect ratio trapping. ${ }^{5,6}$ Furthermore, one may alleviate the lattice mismatch by inserting a buffer layer which in the case of $\operatorname{In}_{\mathrm{x}} \mathrm{Ga}_{0.5-\mathrm{x}} \mathrm{As}_{0.5}$ growth on $\mathrm{Si}$ could be a GaAs buffer. To tailor the crystalline, electrical and optical properties of such nanostructures, one must control the composition precisely.

For many characterization methods, it is intrinsically or practically impossible to reach a spatial resolution $(<1 \mathrm{~nm})$ in line with the dimensions $(2-5 \mathrm{~nm})$ of a single device as

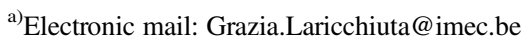

either the probing beam cannot be focused enough and/or the corresponding signal intensity becomes too weak. Yet, as many of the technological processes (growth, dopant incorporation, activation, etc.) are size dependent, the materials characterization needs are shifting from the analysis of (blanket) thin films to the analysis of devices with relevant dimensions. Finally, even if probing only one single device with such spatial resolution would be feasible, the analyzed area and volume become so small that the results might not be representative for the "average" device present on a $300 \mathrm{~mm}$ wafer. To overcome these limitations, one has introduced the concept of ensemble measurements whereby the analysis is performed with a broad beam on an array of nanoscale devices simultaneously. Since these devices have the relevant dimensions, the growth physics can be investigated. Moreover, the summation over many devices provides a good signal-to-noise ratio and captures a statistically relevant amount of devices present on the wafer. By exploiting the underlying physics of the characterization methods, it has been possible to isolate the information of the devices from the surrounding matrix. The study of embedded nanostructures using ensemble measurements has already been reported for small-angle $\mathrm{x}$-ray scattering, for grazing-incidence $\mathrm{x}$-ray fluorescence, ${ }^{7}$ for medium energy ion scattering, ${ }^{8}$ for Raman spectroscopy, ${ }^{9}$ and for secondary ion mass spectrometry. ${ }^{10}$ In the present work, we will present the developments required to extend this approach toward Rutherford backscattering spectrometry (RBS). Consequently, we will demonstrate that RBS can be promoted from the analysis of blanket films to confined devices of relevant dimensions. 
RBS is a quantitative materials characterization method for atoms areal densities and composition depth profiles, ${ }^{11}$ used as a reference for its high traceability. ${ }^{12-14}$ Whereas RBS is commonly applied for wafer-scale characterization of the atomic areal density of thin films ${ }^{15,16}$ and for compositional depth profiling of blanket, layered structures,${ }^{17}$ its application to analyze laterally inhomogeneous samples is largely unexplored. ${ }^{18} \mathrm{~A}$ few attempts have been conducted to use RBS to characterize spherical or ellipsoidal nanoparticles. ${ }^{19,20}$ In those cases, the RBS spectra are analyzed using analytical models specifically developed for the nanoparticles. However, it is difficult to generalize the existing models to analyze nanostructures of complex shapes and concentration depth profiles. Here, we study a periodic ensemble of InGaAs nanostructures embedded in an $\mathrm{SiO}_{2}$ matrix and employ a versatile computational method to analyze the RBS spectra.

\section{EXPERIMENT}

The periodic arrays of nanostructures are obtained using $300 \mathrm{~mm}$ diameter wafer scale optical lithography and processing. On the wafer, the mask provides multiple areas of $1200 \times 600 \mu \mathrm{m}^{2}$ on which the nanostructures are uniformly and periodically repeated. The starting material is an $\mathrm{SiO}_{2}$ layer on an Si substrate, which is wet-etched by tetramethylammonium hydroxide (TMAH). ${ }^{21}$ The etch properties of TMAH create a V-shaped Si surface with two $\{111\}$ facets. The selective growth of (In) GaAs on $\mathrm{Si}$ is achieved by metal organic vapor phase epitaxy using group-V and group-III precursors such as tertiarybutylarsine, trimethylindium, triethylgallium, and trimethylgallium. ${ }^{21}$ Starting from the Si substrate, the fins consist of three layers (Fig. 1): a GaAs layer ( $72 \mathrm{~nm}$ thick), an $\operatorname{In}_{\mathrm{y}} \mathrm{Ga}_{0.5-\mathrm{y}} \mathrm{As}_{0.5}$ layer with a gradual increase of indium content ( $24 \mathrm{~nm}$ thick), and an $\mathrm{In}_{\mathrm{x}} \mathrm{Ga}_{0.5-\mathrm{x}} \mathrm{As}_{0.5}$ layer with constant indium content $(48 \mathrm{~nm}$ thick). Areas with fins of $16 \mathrm{~nm}$ up to $50 \mathrm{~nm}$ surface opening (or width, W) are available.
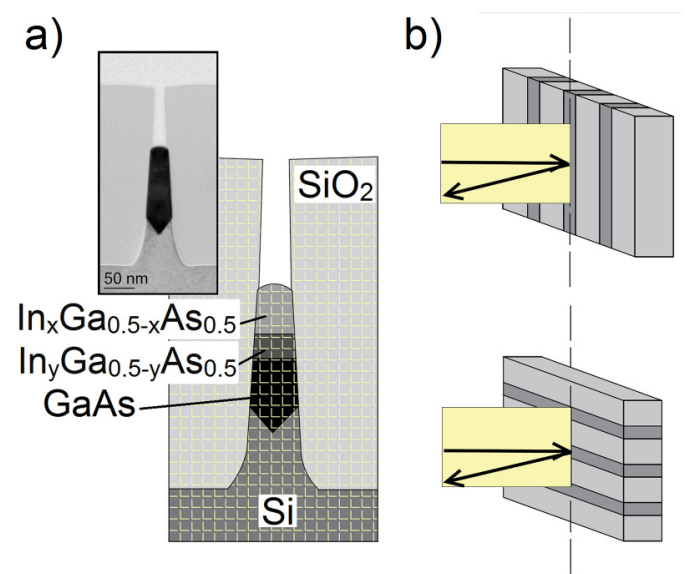

FIg. 1. (a) Pixeled image and TEM cross section (inset) of $20 \mathrm{~nm}$ wide fin made of (In) GaAs in $\mathrm{SiO}_{2}$ trenches on an $\mathrm{Si}$ substrate. The different grayscales refer to possibly different materials and compositions. (b) Measurement geometry with fins parallel (top) and perpendicular (bottom) to the scattering plane. The dashed line represents the sample rotation axis.
The transmission electron microscopy (TEM) images are obtained with a double corrected FEI Titan 60-300 Cube TEM/Scanning TEM tool operating at $120 \mathrm{kV}$, equipped with a superX energy-dispersive $\mathrm{X}$-ray spectroscopy (EDS) system (Bruker). The uncertainty on the dimensions of the fins as derived from the TEM images is estimated as less than one nanometer.

The RBS measurements are performed with a $1.5 \mathrm{MeV}$ ${ }^{4} \mathrm{He}^{+}$beam and a beam current of $20 \mathrm{nA}$. The beam spot is $0.4 \times 0.4 \mathrm{~mm}^{2}$. The detector is a silicon strip detector of $1.79 \mathrm{~mm}$ width and $29 \mathrm{~mm}$ height. ${ }^{22}$ The detector is placed at a scattering angle of $162^{\circ} \pm 0.5^{\circ}$ with the long side of the strip oriented perpendicular to the scattering plane. The detector is placed at a distance of $126 \mathrm{~mm}$ from the sample and the solid angle of the detector is $3 \mathrm{msr}$. The energy calibration and solid angle of the detector are derived from the measurement of a reference sample consisting of $5.73 \times 10^{15}$ bismuth atoms $/ \mathrm{cm}^{2}$ implanted in silicon. ${ }^{23,24}$ The measurements are performed with the ion beam incident at $2^{\circ}$ off the sample normal both in the horizontal and in the vertical direction.

\section{RESULTS AND DISCUSSION}

Focusing on the interpretation of the RBS spectra, one must consider the known working principles of RBS (kinematic factor, scattering cross section, and energy $\operatorname{loss}^{11}$ ). Firstly, because of the kinematic factor, the signal of the heavy elements in the fins (In, Ga, As) in the RBS spectra is well-separated from the signal of the surrounding matrix $\left(\mathrm{Si}, \mathrm{SiO}_{2}\right)$. It is not possible to discriminate the signals from Ga and As in the RBS spectra. Yet, it is commonly accepted for epitaxial (In)GaAs growth that charge neutrality applies, and thus the As content equals the sum of the In content plus the Ga content. We assume charge neutrality for the InGaAs compound to interpret the RBS spectra. Alternatively, one could use high energy primary beams, e.g., $>4 \mathrm{MeV}^{4} \mathrm{He}^{+}$, and high resolution RBS (Ref. 25) to discriminate the $\mathrm{Ga}$ and As signals, or derive the $\mathrm{Ga} / \mathrm{As}$ ratio from particle induced $\mathrm{x}$-ray emission. Secondly, the integrated signal of the elements in the fins is proportional to the mean areal coverage of the fins and their composition. Thirdly, the energy dependence of the signal is influenced by the materials traversed along the in- and outgoing ion paths. The latter poses additional challenges in the case of embedded fins as compared to the cases of blanket films and free standing nanostructures. ${ }^{26}$ A stochastic approach, as proposed in Ref. 27 and implemented, for example, in Ref. 28 , is well-suited to analyze RBS spectra on samples containing non periodically or randomly distributed embedded nanostructures. ${ }^{29}$ In the case of periodically repeated embedded fins, the in- and outward trajectories depend on the impact point of the incident particle and on the depth of the collision. Hence, each backscattered particle may have traversed different layer stacks on their inward and outward path. This is illustrated in Fig. 2(a) where particles experiencing a collision at different depths will traverse different sections of the fin as well as of the $\mathrm{SiO}_{2}$ matrix on their trajectory toward the detector. 


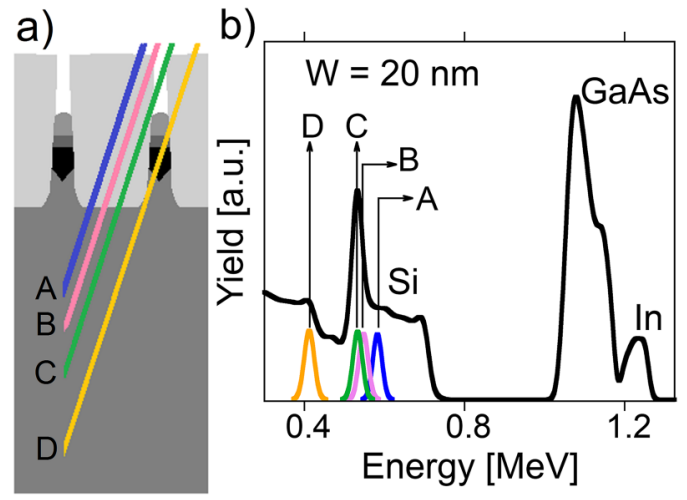

FIG. 2. (a) Simulated ion trajectories (continuous lines) for backscattering at four depths (A, B, C and D) and with the incidence in the center of the fin. (b) Simulated RBS sub-spectrum from ions incident in the fin center and backscattering at all depths. Simulated RBS signals (peaks A, B, C and D) [colors and letters relate to (a)] for all trajectories of (a). The primary beam of the simulation is $1.5 \mathrm{MeV}^{4} \mathrm{He}^{+}$, the scattering and incidence angles are $162^{\circ}$ and $0^{\circ}$, respectively.

Different from the case of RBS spectra on planar systems, for the interpretation of RBS spectra on fins, one must also take into account the orientation of the fins with respect to the scattering plane. As two extreme situations, we consider the fins either along the scattering plane or perpendicular to it [respectively, Fig. 1(b) top and bottom]. If the fins were perfectly rectangular and if the detector was ideally small, then the modeling of the spectra in the parallel geometry could be reduced to the superposition of two planar substructures. However, the fins are not perfectly rectangular and show a vertical recess compared to the surrounding matrix, and the detector has a finite size. We calculated that in the parallel geometry and for fins as depicted in Fig. 1(a), 89\% of the backscattering particles from the fins do traverse a section of the $\mathrm{SiO}_{2}$ matrix. In the perpendicular geometry, all of the particles backscattered from the fins do traverse a section of the $\mathrm{SiO}_{2}$ matrix. Therefore, in both the parallel and perpendicular geometry, ions backscattering from the fins traverse many different materials in both the vertical and lateral directions. In this work, we have chosen to perform the experiments in the perpendicular geometry, since then the scattering plane coincides with the cross section of the fins and all the ion paths can be represented in a single two-dimensional representation [Fig. 2(a)].

To arrive at a quantitative interpretation of the RBS spectra, one thus must model the energy losses correctly using the detailed shape of the nanostructures as an input. In such a simulation, the shape of the nanostructure is used to calculate the trajectories, the corresponding energy losses and the final energy seen by the detector for a multitude of He-atoms incident on the nanostructure and on the matrix. Depending on where the He-atom was backscattering within the sample and depending on its incidence position along the surface, it will traverse on its ingoing and outgoing path a different stack of materials or a void in the case of fins shorter than the matrix and thus be subjected to a different energy loss. To account for the variety of scattering paths, we employ the code StructNRA (Ref. 30 and 31) to compute quasirandom incidence points (i.e., numbers with low discrepancy by additive recurrence), backscattering events, trajectories and thus final energies, such that their sum allows to create a complete RBS spectrum.

In the StructNRA program, the sample is represented by a pixel image with adjustable pixel size $\Delta$, each pixel containing a specific material. The pixel image of a $20 \mathrm{~nm}$ wide nanostructure is shown in Fig. 1(a). The shape of the nanostructure is derived from a high-resolution TEM analysis [inset of Fig. 1(a)]. The nanostructure is composed of three different layers which relate to three different indium concentrations. The section of the fin with a gradual increase of the indium concentration $\left(\operatorname{In}_{\mathrm{y}} \mathrm{Ga}_{0.5-\mathrm{y}} \mathrm{As}_{0.5}\right)$ is modeled as a layer with indium concentration being the average of the indium concentrations of the top and bottom sections. As the indium concentrations are a priori unknown, their exact values are optimized through the comparison of the simulated spectra with the experimental spectra. A simulated StructNRA spectrum is obtained by integrating 1000 sub-RBS-spectra, each one being the collection of multiple ions incident at the same point on the surface and backscattering at different depths. In Fig. 2(b), we show the sub-RBS-spectrum for particles incident at the center of the fin with the backscattering plane perpendicular to the fins. The final energies of such particles are numerically calculated using the energies lost in each traversed pixel. Since the geometrical and compositional properties of the (pixels within the) nanostructures affect the energy loss of the backscattered particles, one needs to consider the stopping power of the material traversed along the trajectories and to calculate the ion path length in each pixel. For example, the stopping power is high when the particle is inside the InGaAs material, reduced when traversing the $\mathrm{SiO}_{2}$ and zero in the empty space on top of the fins [see Fig. 2(a)]. The stopping power encountered in each pixel is determined with the Ziegler and Biersack model ${ }^{32}$ according to the material contained in the pixel. StructNRA calculates the intersection points between the trajectory of the particle and the pixel grid of the image and then it evaluates the path length within the pixel using the pixel size (expressed in nanometer) and the mass density of each compound in the target. The mass density of $\mathrm{SiO}_{2}$ in the trenches is $2.65 \mathrm{~g} / \mathrm{cm}^{3}$, as it has crystallized during the thermal step at $580{ }^{\circ} \mathrm{C}$ after the GaAs deposition. ${ }^{21}$ For GaAs, the mass density of $5.32 \mathrm{~g} / \mathrm{cm}^{3}$ is adopted. For $\mathrm{In}_{\mathrm{x}} \mathrm{Ga}_{0.5-\mathrm{x}} \mathrm{As}_{0.5}$ and $\mathrm{In}_{\mathrm{y}} \mathrm{Ga}_{0.5-\mathrm{y}} \mathrm{As}_{0.5}$, the mass density is the weighted average of the elemental mass densities. The energy loss straggling is calculated including the corrections by Yang et al. ${ }^{33}$ and $\mathrm{Chu}^{34}$ to Bohr's theory. ${ }^{35}$

Using the procedure implemented in StructNRA, one may construct a complete spectrum considering the incidence of $\mathrm{He}$-atoms in all possible positions (minimum step size $=\Delta$ ).

Firstly, we investigate the dependency of the RBS spectrum on the fin shape. In Fig. 3, we compare the spectra simulated for perfectly rectangular fins (thick line) and realistic $20 \mathrm{~nm}$ wide fins (thin line) with equal compositions. The two shapes have the same surface opening and fin volume, while the rectangle height is rescaled to meet the volume requirements. One observes that the RBS spectrum of the rectangular fins displays a steeper leading edge of the indium 


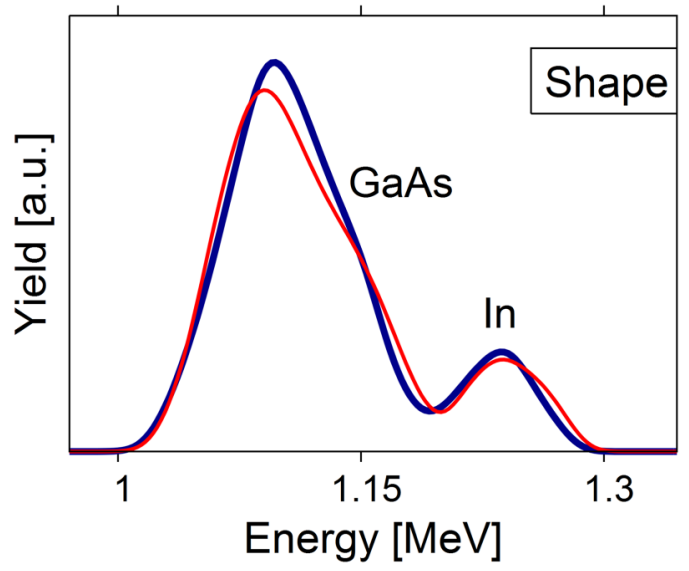

FIG. 3. Simulated spectrum of the In, Ga, and As signals for rectangular fins (thick blue line) and realistic $20 \mathrm{~nm}$ fins (thin red line) in the perpendicular geometry.

and gallium-arsenic signals. These observations illustrate that the detailed shape of the nanostructures needs to be taken into account to analyze the experimental RBS spectra or, vice versa, that the spectra contain information on the details of the fin shape.

Secondly, we consider the effect on the RBS spectra of a potential diffusion of indium toward the bottom interface. It is recognized that the signal in the RBS spectra from excess indium at the bottom interface overlaps with the intense signal from $\mathrm{Ga}$ and As. The effect of a realistic variation (up to 5 at. \%) of the indium concentration at the bottom interface cannot be discerned in the present spectra. Conversely, a potential segregation of indium at the bottom interface does not affect the quantification of the indium concentration in the top section of the fins.

Thirdly, we investigate the dependency of the RBS spectra on the mean areal coverage of the fins. We compare the simulated RBS spectra for 20 and $21 \mathrm{~nm}$ wide fins. The modeling assumes that the various fins all have the same mean realistic shape and mean concentration profile. Variations of the compositions within the fins, if not systematic, are averaged out and do not affect the spectra.

In the fin layers containing indium, the mean areal coverage of the $21 \mathrm{~nm}$ fins is $3 \%$ larger than the coverage of the $20 \mathrm{~nm}$ fins. Accordingly, the integrated indium signal from the $21 \mathrm{~nm}$ fins is $3 \%$ more than the signal from the $20 \mathrm{~nm}$ fins. A $1 \%$ combined standard uncertainty can be reached with RBS for the composition determination of blanket films. ${ }^{12,36,37}$ On the other hand, for confined nanostructures, the composition and the areal coverage are correlated parameters as they both affect the indium signal height. Based on the above, we believe the technique to be sensitive to a variation of $1 \mathrm{~nm}$ of the mean width of the fins in the ensemble.

In Fig. 4, we compare the experimental RBS spectra for samples containing fins of 16 and $50 \mathrm{~nm}$, respectively. Due to the larger areal coverage, the In signal for the wide fins $(\mathrm{W}=50 \mathrm{~nm})$ should be more intense (3.1 times) as compared to the narrow fins $(\mathrm{W}=16 \mathrm{~nm})$. However, an even larger increase (3.4 times) of the In signal is observed as a result of a larger In content in the wide fins $(x=0.20 \pm 0.01)$ a)
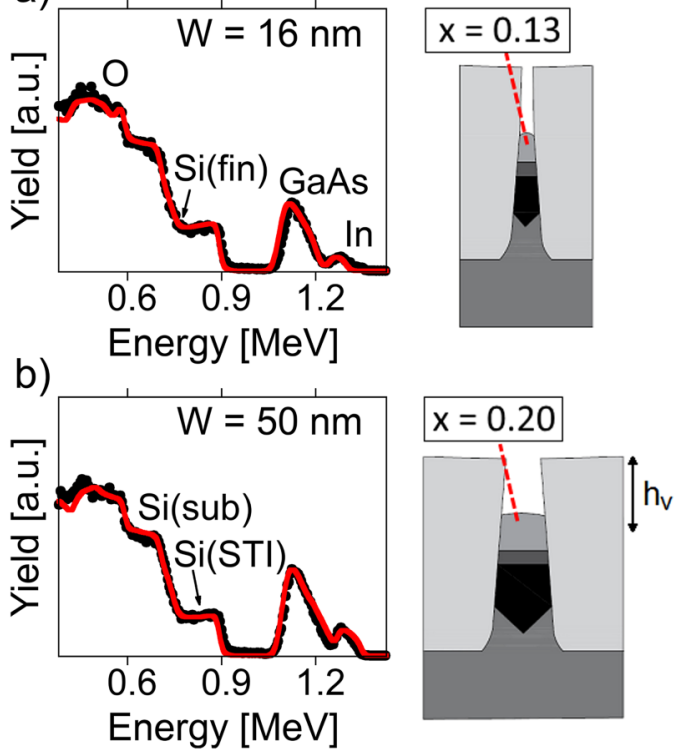

FIG. 4. Left: RBS spectra (dots) for $1.5 \mathrm{MeV}{ }^{4} \mathrm{He}^{+}$ions and a scattering angle of $162^{\circ}$. The continuous lines are best fit simulations. Right: indium content in the top layer of (a) $16 \mathrm{~nm}$ and (b) $50 \mathrm{~nm}$ wide fins.

versus the narrow fins $(x=0.13 \pm 0.01)$. The reported uncertainties are dominated by the counting statistics and the uncertainty on the optimization of the simulated spectra with respect to the experimental spectra.

A detailed analysis of the size dependence ${ }^{38}$ indicates that the relevant parameter is not the width but rather the aspect ratio of the void in which the growth occurs, i.e., $h_{v} / \mathrm{W}$, where $\mathrm{h}_{\mathrm{v}}$ and $\mathrm{W}$ are the height and the width of the void, respectively [Fig. 4(b)]. In Fig. 5, one observes a linear relationship between the reciprocal of the indium concentration $(1 / x)$ and the aspect ratio of the void. This is understood by realizing that the growth rate of InAs and of GaAs in narrow trenches is dominated by the Knudsen diffusion such that the reciprocals of the growth rate of GaAs and InAs are expected to linearly depend on the aspect ratio. ${ }^{38}$ The observed linear relationship between the reciprocal of the indium concentration and the aspect ratio (continuous curve in Fig. 5) indicates that

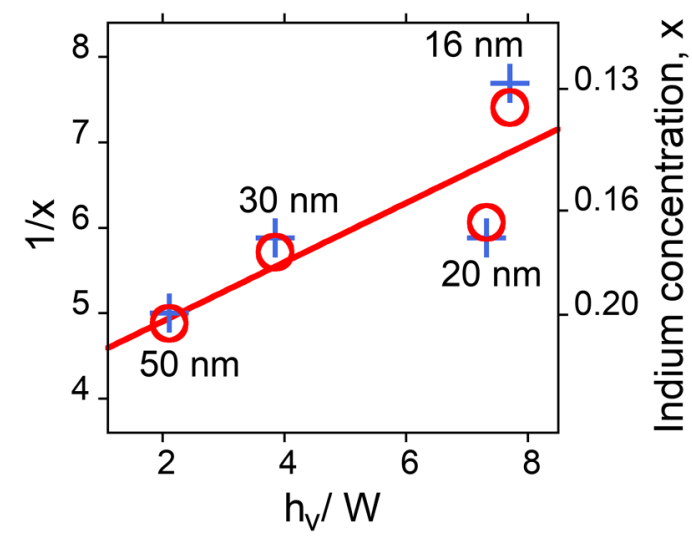

FIG. 5. Indium concentration in the top layer of the fin as a function of the aspect ratio of the void. Results are obtained through RBS (circles) and TEM-EDS (crosses) and are modeled by a linear function (continuous line). 
the variable InAs growth rate as a function of width is the dominating process determining the composition.

To further confirm the experimental results, we also performed a detailed composition analysis of individual fins using TEM-EDS. The TEM data points in Fig. 5 (crosses) represent the average composition along the central section of the top layer of one fin and are in close agreement with the concentrations from RBS (circles) which refer to the average composition of the top layers of a large ensemble of fins.

\section{CONCLUSIONS}

In conclusion, we demonstrate the application of RBS for the analysis of periodically repeated nanostructures embedded in an $\mathrm{SiO}_{2}$ matrix. Through the ensemble measurements, one obtains enough sensitivity to probe composition variations as well as size variations. By incorporating the detailed shape of the nanostructures into the simulation of the RBS spectra, a quantitative interpretation becomes possible. We identify the dependence of the In concentration on the fin width, and we explain it to originate from the size dependent growth rate of InAs.

It is anticipated that implementing multiple detectors at various geometries will allow one to arrive at a depth and lateral-resolved composition profile from the RBS spectra.

\section{ACKNOWLEDGMENTS}

P. Favia and O. Richard (imec) are acknowledged for the TEM-EDS analysis. B. Kunert (imec) and A. Schulze (Applied Materials Inc.) are acknowledged for sample fabrication and scientific discussions. Stimulating discussions with $\mathrm{H}$. Bender and P. van der Heide (imec) are greatly appreciated.

${ }^{1}$ M. Heyns, M. Meuris, and M. Caymax, ECS Trans. 3, 511 (2006).

${ }^{2}$ A. McKee, C. J. McLean, G. Lullo, A. C. Bryce, R. M. De La Rue, J. H. Marsh, and C. C. Button, IEEE J. Quantum Electron. 33, 45 (1997).

${ }^{3}$ V. M. Ustinov et al., Appl. Phys. Lett. 74, 2815 (1999).

${ }^{4}$ S. Liu, D. Jung, J. C. Norman, M. J. Kennedy, A. C. Gossard, and J. E. Bowers, Electron. Lett. 54, 432 (2018).

${ }^{5}$ E. A. Fitzgerald and N. Chand, J. Electron. Mater. 20, 839 (1991).

${ }^{6}$ J. Z. Li, J. Bai, C. Major, M. Carroll, A. Lochtefeld, and Z. Shellenbarger, J. Appl. Phys. 103, 106102 (2008).

${ }^{7}$ V. Soltwisch, P. Hönicke, Y. Kayser, J. Eilbracht, J. Probst, F. Scholze, and B. Beckhoff, Nanoscale 10, 6177 (2018).
${ }^{8}$ J. England, W. Möller, J. A. van den Berg, A. Rossall, W. J. Min, and J. Kim, Nucl. Instrum. Methods B 409, 60 (2017).

${ }^{9}$ T. Nuytten, J. Bogdanowicz, T. Hantschel, A. Schulze, P. Favia, H. Bender, I. De Wolf, and W. Vandervorst, Adv. Eng. Mater. 19, 8 (2017).

${ }^{10}$ A. Franquet, B. Douhard, D. Melkonyan, P. Favia, T. Conard, and W. Vandervorst, Appl. Surf. Sci. 365, 143 (2016).

${ }^{11}$ W. K. Chu, J. W. Mayer, and M. A. Nicolet, Backscattering Spectrometry (Academic, New York, 1978).

${ }^{12}$ C. Jeynes, N. P. Barradas, and E. Szilágyi, Anal. Chem. 84, 6061 (2012).

${ }^{13}$ D. J. Dunstan et al., J. Cryst. Growth 126, 589 (1993).

${ }^{14}$ C. Jeynes, Z. H. Jafri, R. P. Webb, A. C. Kimber, and M. J. Ashwin, Surf. Interface Anal. 25, 254 (1997).

${ }^{15}$ J. Meersschaut and W. Vandervorst, Nucl. Instrum. Methods B 406, 25 (2017).

${ }^{16}$ G. Laricchiuta, W. Vandervorst, I. Zyulkov, S. Armini, and J. Meersschaut, J. Vac. Sci. Technol. A 36, 02 D407 (2018).

${ }^{17}$ C. Jeynes and G. L. Colaux, Analyst 141, 5944 (2016).

${ }^{18}$ S. R. C. Pinto, R. J. Kashtiban, A. G. Rolo, M. Buljan, A. Chahboun, U. Bangert, N. P. Barradas, E. Alves, and M. J. M. Gomes, Thin Solid Films 518, 2569 (2010).

${ }^{19}$ M. I. Ortiz, J. Sangrador, A. Rodríguez, T. Rodríguez, A. Kling, N. Franco, N. P. Barradas, and C. Ballesteros, Phys. Status Solidi A 203, 1284 (2006).

${ }^{20}$ Z. Zolnai, N. Nagy, A. Deák, G. Battistig, and E. Kótai, Phys. Rev. B 83, 233302 (2011).

${ }^{21}$ W. Guo, Y. Mols, J. Belz, A. Beyer, K. Volz, A. Schulze, R. Langer, and B. Kunert, J. Appl. Phys. 122, 025303 (2017).

${ }^{22}$ V. Alarcon-Diez, I. Vickridge, M. Jakšić, V. Grilj, B. Schmidt, and H. Lange, Nucl. Instrum. Methods B 406, 148 (2017).

${ }^{23}$ U. Wätjen and H. Bax, Nucl. Instrum. Methods B 85, 627 (1994).

${ }^{24}$ C. Cohen, J. A. Davies, A. V. Drigo, and T. E. Jackman, Nucl. Instrum. Methods 218, 147 (1983).

${ }^{25}$ W. G. Skala, A. W. Haberl, H. Bakhru, and W. Lanford, AIP Conf. Proc. 1525, 199 (2013).

${ }^{26}$ H. Langhuth, M. Mayer, and S. Lindig, Nucl. Instrum. Methods B 269, 1811 (2011).

${ }^{27}$ J. P. Stoquert and T. Szörényi, Phys. Rev. B 66, 144108 (2002).

${ }^{28}$ N. P. Barradas and C. Jeynes, Nucl. Instrum. Methods B 266, 1875 (2008).

${ }^{29}$ P. Guha, J. R. Rao, and P. V. Satyam, Nucl. Instrum. Methods B 409, 209 (2017).

${ }^{30}$ M. Mayer, Nucl. Instrum. Methods B 371, 90 (2016).

${ }^{31}$ M. Mayer, P. Malinský, F. Schiettekatte, and Z. Zolnai, Nucl. Instrum. Methods B 385, 65 (2016).

${ }^{32}$ J. F. Ziegler, J. P. Biersack, and U. Littmark, "The stopping and range of ions in solids," The Stopping and Ranges of Ions in Matter (Pergamon, New York, 1985), Vol. 1.

${ }^{33}$ Q. Yang, D. J. O'Connor, and Z. Wang, Nucl. Instrum. Methods B 61, 149 (1991).

${ }^{34}$ W. K. Chu, Phys. Rev. A 13, 2057 (1976).

${ }^{35}$ N. Bohr, Mat. Fys. Medd. Dan. Vid. Selsk. 18, 1 (1948).

${ }^{36}$ G. L. Colaux and C. Jeynes, Anal. Methods 6, 120 (2014).

${ }^{37}$ C. Jeynes, Nucl. Instrum. Methods B 406, 30 (2017).

${ }^{38}$ S. Jiang, C. Merckling, W. Guo, N. Waldron, M. Caymax, W. Vandervorst, M. Seefeldt, and M. Heyns, J. Cryst. Growth 391, 59 (2014). 